\title{
Discussion on Misclassification of Ultrasonic Cross-hole Detection Method in Bored Piles
}

\author{
Likui Huang ${ }^{1, a}$, Enkuan Tang ${ }^{2, b}$, Ganming $\mathrm{Yi}^{2, \mathrm{c}}$, \\ Wenbin $\mathrm{Wu}^{1, \mathrm{~d}}$, Qing Shen ${ }^{1, \mathrm{e}}$ \\ ${ }^{1}$ College of Civil Engineering, Hunan University, China \\ ${ }^{2}$ China Construction Fifth Engineering Bureau Co. LTD \\ ahuanglikui@hnu.edu.cn, btangenkuan@cscec.dz, ${ }^{C}$ yiganming@cscec.dz, \\ 'wuwenbin@hnu.edu.cn, ${ }^{\circledR}$ shenging@hnu.edu.cn
}

Keywords: Cross-hole detection method; Bored pile; Misjudgment

\begin{abstract}
Ultrasonic cross-hole detection method is widely used for the quality detection of bored piles due to its comprehensive, detailed and accurate results, and no restrictions on the lengths and ratios of length to diameter of piles. However, because of the quality problems of the instrument or operation rather than the quality of the pile itself, the ultrasonic testing results are not good enough, and the misjudgment often occurs. The paper analyzed the characteristics and the causes of the problems, and discussed how to identify and reduce the occurrence of misjudgment by summarizing the experiences from the ultrasonic testing results of the 5,000 piles for the $53 \mathrm{~km}$ North-South Expressway project in Algeria, and combining relevant specifications and literatures.
\end{abstract}

\section{Introduction}

Bored piles are widely used in highway, railway, industrial and civil building, water conservancy and hydropower, and port engineering. The usual methods for quality detection of bored piles include static load method, low-high strain dynamic test method, core drilling method, and ultrasonic cross-hole detection method. Compared with the static load method and the high strain method, the ultrasonic cross-hole detection method has the advantages of simple operation and low cost. Compared with the low-strain method, the detection results of ultrasonic cross-hole detection method are meticulous and intuitive. Especially, the exact position and size of the defects can be measured in this way. Compared with the core drilling method, ultrasonic cross-hole detection method is more comprehensive, and can cover almost the entire section of the pile foundation. With the maturity of technology and the update of instruments and software, the ultrasonic cross-hole detection method is more convenient to operate, data processing is faster, and it is favored in modern engineering.

The basic principle of ultrasonic cross-hole testing of bored piles is to place ultrasonic emission and receiving transducers separately in the pre-embedded acoustic tubes. Water is generally used as a coupling agent. The acoustic parameters, such as wave speed, amplitude and frequency of ultrasonic waves penetrating the concrete body between two acoustic tubes are measured to observe the relative changes of parameters to determine the integrity and defects of the pile foundation concrete. As a semi-indirect quality detection method, it requires a relatively high level of basic theory and operating experience for detection personnel. There are 6,863 bridge piles for the North-South Expressway project in Algeria. In order to ensure the progress and timely reflect the actual quality condition of the piles, all of the piles are permitted to test by the general contractor, which has been negotiated with the project owner and supervisor. Due to the changeable conditions in the test site, it is easy to cause misjudgment of the test results. This paper discusses several common error-prone judgments in quality detection of bored piles using ultrasonic cross-hole method.

\section{Lack of coupling agent}

Before entering the concrete body, ultrasonic waves emitted from the transducer must propagate and couple with a couplant as the medium, to reduce the energy loss due to incidence and reflection of ultrasonic waves. On the one hand, more ultrasonic energy is introduced into the measured medium, and on the other hand, ultrasonic signals transmitted through the concrete body are received as much as possible by the test system. In the cross-hole detection, clean water is 
generally used as the coupling agent between the transducer and the concrete. Sometimes, the top part of a single or several acoustic tubes is not full of the coupling agent due to human negligence or poor sealing of the acoustic tubes. When there is no the coupling agent, the ultrasonic wave emitted by the transmitting transducer does not penetrate the concrete body very well, and the ultrasonic energy is greatly weakened. The detection results are shown as energy blank, and the amplitude is small or no wave, as shown in figure 1.

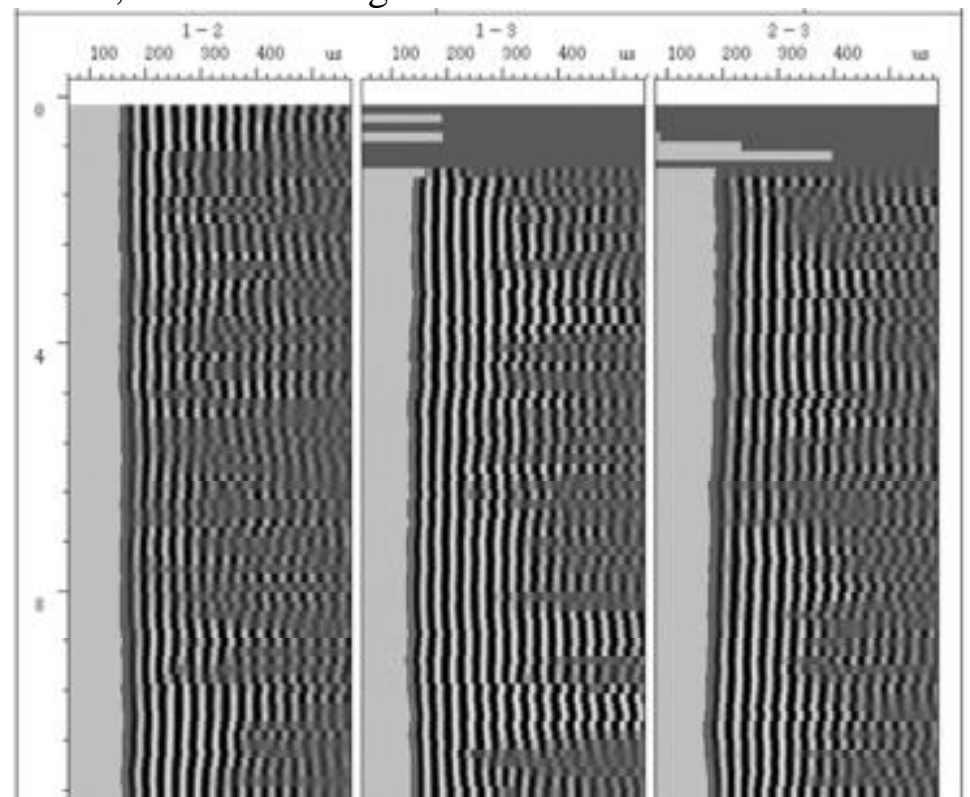

Figure 1 Grayscale at lack of couplant in part acoustic tube

Acoustic tube will affect several sections associated with it. If these sections start at the same location, the amplitude is sharply reduced, and the energy becomes blank until the top of the pile, the reason is probably not the problem of the pile itself, it is the acoustic tube coupling agent that is not full and the couplant should be checked.

\section{Acoustic tube bending}

In the process of making and hoisting the pile foundation steel cage and pouring the concrete, the acoustic tube may be bent. Acoustic tube bend will change the distance between the two acoustic tubes, the first wave advance or lag, which affects the calculation of the wave speed and has appearance of abnormal wave speed area, as shown in figure 2.

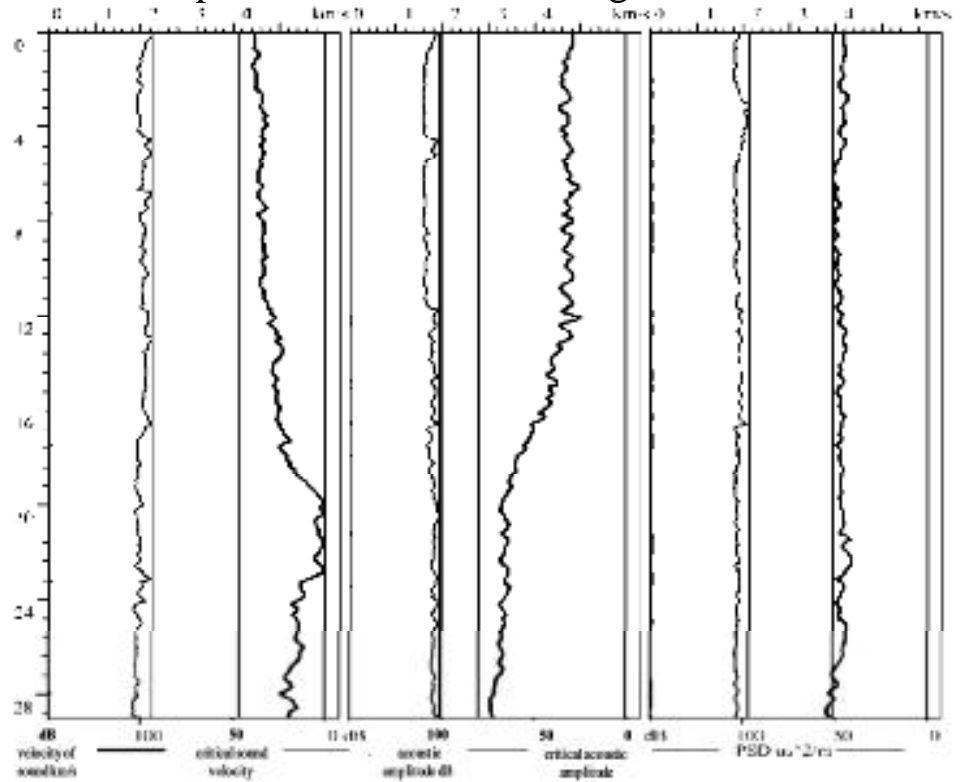

Figure $2 \mathrm{~V}-\mathrm{H}, \mathrm{A}-\mathrm{H}$, and PSD lines when the acoustic tube bends

The variation of wave velocity caused by the bend of acoustic tube will affect many sections 
related to the tube under normal circumstances, because the curvature radius of the tube is large, the velocity of sound will change slowly with the depth of the tube and will not change suddenly. The bending of the acoustic tube does not change the amplitude of the wave significantly. In order to eliminate the influence of the bend of acoustic tube, the results should be determined by combining the PSD curve.

$$
P S D=\frac{t_{\mathrm{i}}-t_{\mathrm{i}-1}}{z_{\mathrm{i}}-z_{\mathrm{i}-1}}\left(t_{\mathrm{i}}-t_{\mathrm{i}-1}\right)
$$

Where: $t_{\mathrm{i}}, t_{\mathrm{i}-1}$ are the sonic times at the $i$ and $i-1$ measurement points, respectively.

$z_{\mathrm{i}}, z_{\mathrm{i}-1}$ are the depths of the $i$ and $i-1$ measurement points, respectively.

\section{Receiving and emission transducers at different horizontal positions during detection}

According to the change of relative height of transducers, the cross-hole detection can be divided into several types: horizontal, oblique, cross oblique, and sector scanning measurement, etc. However, the horizontal measurement is the main method, and the other methods are chosen only after the anomaly of the horizontal measurement result.

The horizontal measurement requires the two transducers always to be at the same height during the lifting process, as shown in figure 3(a). The inconsistent heights of transducers before detection or during hoisting are shown in figure 3(b).

When the relative height of the transducer is different, the horizontal measurement method has actually become oblique, and the distance between the two transducers has become longer, so the measured sound velocity will be smaller than actual sound velocity. The received energy angle of the transducer becomes smaller, so the measured waveform will be weaker than the flat measurement method. This is pretty similar to the symptoms when the concrete is not dense enough, the tester should carefully check the depth mark on the cable before starting the test, so that the transducer will always be in a flat state, and if there are any suspicious defects, it should be tested again after the completion of the leveling test.

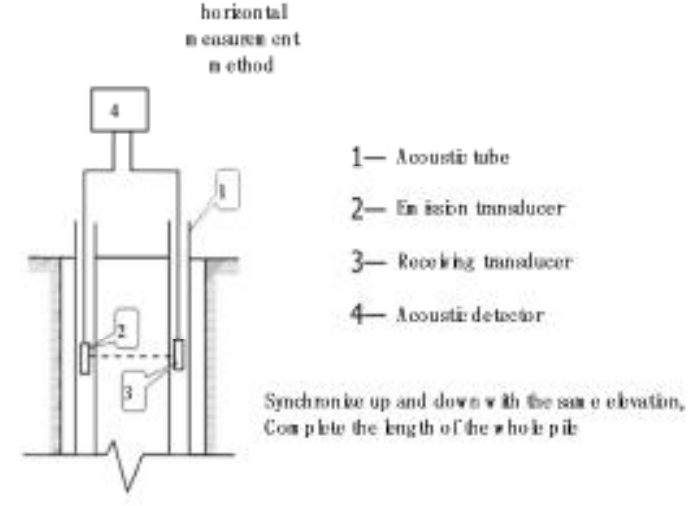

Figure 3(a) Transducers at the same height

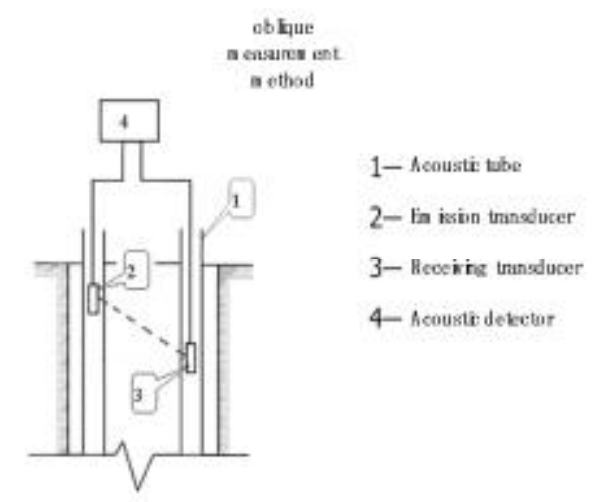

Figure 3(b) Transducers at the different heights

\section{Failure of centralizer}

In order to enable transducers to enter the acoustic tube and move freely up and down in the acoustic tube, the Technical Specification for Dynamic Measurement of Pile Foundation in Highway Engineering (JTG/T F8-01-2004) demanded that "the inner diameter of acoustic measuring tube should be larger than the outer diameter of transducer", so it is necessary to install fixed transducer and centralizer with relative distance of pipe wall on the transducer.

When the detector is used for a long time, the locating piece is worn out or the acoustic tube does not match the probe diameter. It can cause the centralizer to fail, and the transducer can swing freely in the acoustic tube. The results are follows: first, the span changes continuously with the swing of transducer, which causes the detected waveforms to be arranged in a discontinuous order, and the first waves are sometimes in advance and then lag behind. Second, the horizontal inclination of ultrasonic emission also has a small change, when the receiving transducer is not in the receiving transducer is not in the direction of ultrasonic emission, the received waveform naturally weakens. Therefore, the ultrasonic parameters are chaotic, as shown in figure 4. 
It is easy to calculate the maximum acoustic variation $\Delta t$ caused by free oscillation of the transducer if there is no centralizer. Taking the sounding pipe with an internal diameter of $D=60$ $\mathrm{mm}$ and the probe with an external diameter of $d=25 \mathrm{~mm}$ as example, if the wave velocity of ultrasonic wave in water is $v=1.5 \mathrm{~km} / \mathrm{s}$, then $\Delta t=(D-d) / v=(60 \mathrm{~mm}-25 \mathrm{~mm}) /(1.5 \mathrm{~km} / \mathrm{s}) \approx 23.3 \mu \mathrm{s}$. This result is basically consistent with the duration of wave train in the figure above, and it is also in the same order of magnitude as the vibration period of the supersonic wave used, so its influence cannot be ignored.

In order to avoid the transducer's influence of oscillation, the diameter of the selected sounding pipe should not be too large, and the minimum size for the transducer to be smoothly placed is appropriate. In addition, the worn centralizer should be replaced regularly, as the damping effect of a rubber centralizer also has certain benefits for the maintenance of transducer.
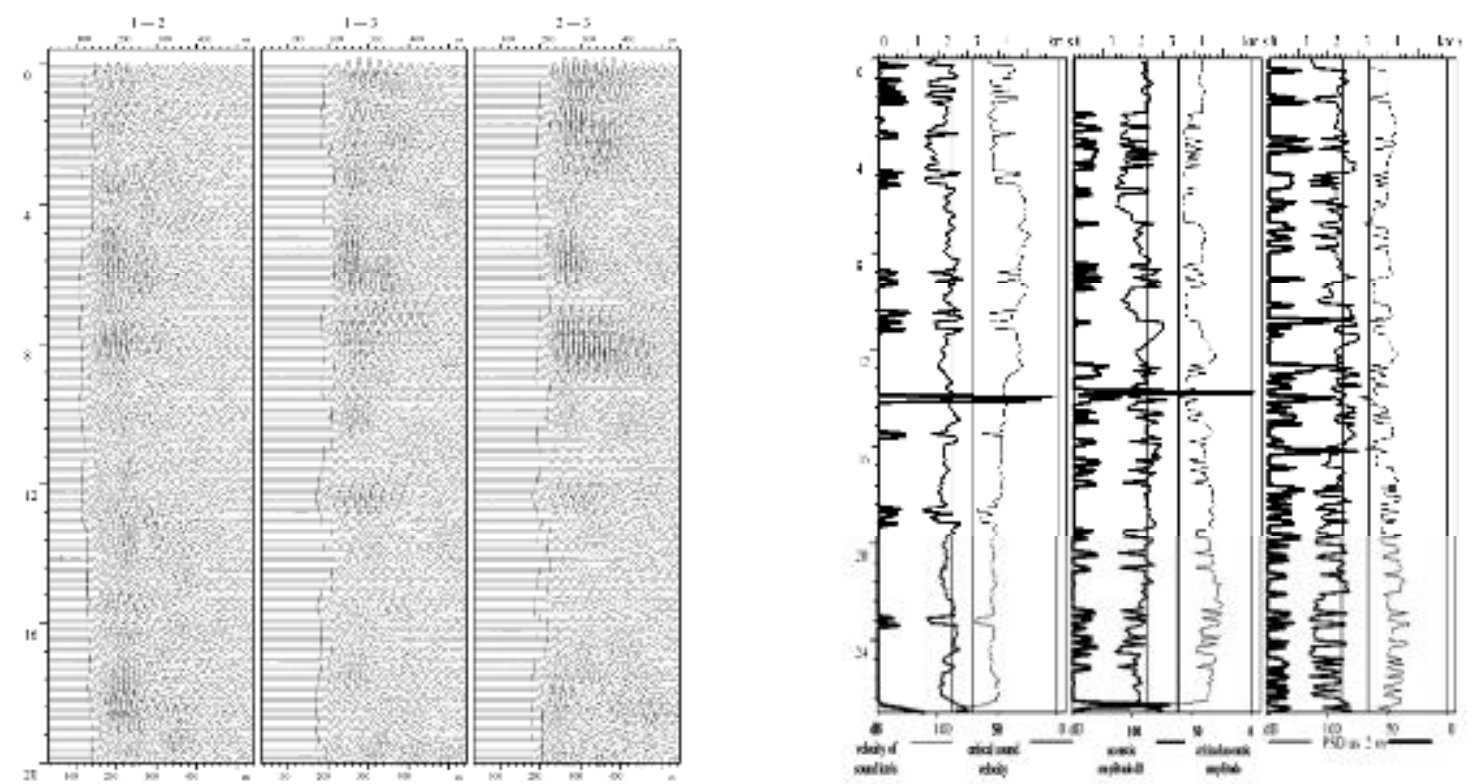

Figure 4 Wave train figure (left), and $V-H, A-H$, and PSD curves (right) at failure of centralizer

\section{Conclusions}

Although the ultrasonic cross-hole method for testing pile foundation is relatively intuitive and accurate, it is after all a semi-indirect method. Simple conclusions based on a single test method will inevitably lead to inaccurate results. This requires us to accumulate experience and improve professional knowledge. When faced with suspected defects, we should consider them from various aspects and eliminate possible interference factors, obtaining test results carefully based on core drilling method and other testing modes so that unnecessary engineering losses can be avoided.

\section{References:}

[1] JTG/T F81-01-2004: Technical Specification of Dynamic Pile Tests for Highway Engineering [S].

[2] CECS 21:2000: Technical Specification for Inspection of Concrete Defects by Ultrasonic Method [S].

[3] F. Chen, T. Xu, J. Chen, L. Guan: Inspection Technology for Foundation Pile Quality (Second edition)[M], China Building Industry Press, 2014.

[4] T. Liu, Z. Zhao, H. Wu: Foundation Pile Detection Technology and Examples [M], China Building Industry Press, 2006. 